\title{
Extracorporeal shock wave therapy combined with engineered mesenchymal stem cells expressing stromal cell-derived factor-1 can improve erectile dysfunction in streptozotocin-induced diabetic rats
}

\author{
Dongho Shin $^{1 \wedge}$, Seung Hwan Jeon ${ }^{1,2}$, Wen Jie Tian², Eun Bi Kwon², Ga Eun Kim², Woong Jin Bae ${ }^{1}$, \\ Hyuk Jin Cho ${ }^{1}$, Sung-Hoo Hong ${ }^{1}$, Ji Youl Lee ${ }^{1}$, Sae Woong Kim ${ }^{1,2}$ \\ ${ }^{1}$ Department of Urology, Seoul St. Mary's Hospital, College of Medicine, The Catholic University of Korea, Seoul, Republic of Korea; ${ }^{2}$ Catholic \\ Integrative Medicine Research Institute, The Catholic University of Korea, Seoul, Korea \\ Contributions: (I) Conception and design: D Shin, SH Jeon, SW Kim; (II) Administrative support: D Shin, SH Jeon, SW Kim; (III) Provision of study \\ materials or patients: All authors; (IV) Collection and assembly of data: All authors; (V) Data analysis and interpretation: D Shin, SH Jeon, SW Kim; \\ (VI) Manuscript writing: All authors; (VII) Final approval of manuscript: All authors. \\ Correspondence to: Sae Woong Kim, MD, PhD. Department of Urology, Seoul St. Mary's Hospital, College of Medicine, The Catholic University of \\ Korea, 222, Banpo-daero, Seocho-gu, Seoul, 06591, Republic of Korea. Email: ksw1227@catholic.ac.kr.
}

Background: For erectile dysfunction (ED) in diabetes mellitus (DM) patients who have poor response to
drugs, extracorporeal shock wave therapy (ESWT) and engineered mesenchymal stem cell (MSC) therapy
have been studied as alternative treatment options. The objective of this study is to investigate whether
ESWT in combination with stromal cell-derived factor-1 expressing engineered mesenchymal stem cell
(SDF-1 eMSC) therapy can have synergistic effects on ED in streptozotocin-induced diabetic rats.
Methods: Fifty 8 -week-old male Sprague-Dawley rats were randomly divided into five groups (N=10 per
group): (I) Normal group, (II) DM ED, (III) DM ED + ESWT group, (IV) DM ED + SDF-1 eMSC group,
and (V) DM ED + ESWT + SDF-1 eMSC group. Each groups were treated with bilateral injections of SDF-
1 eMSC or ESWT following the experiment protocol for eight weeks.
Results: The ratio of ICP/MAP was distinctly higher in the DM ED + ESWT + SDF-1 eMSC group than
that in the DM ED group. Concentration of $\alpha$-smooth muscle actin ( $\alpha$-SMA) was elevated the highest in the
DM ED + ESWT + SDF-1 eMSC group. Additionally, ESWT increased the intensity of SDF-1 expression
in the corpus cavernosum. ESWT + SDF-1 eMSC treatment also induced neuronal nitric oxide synthase
(nNOS) and NO/cGMP expression in the corpus cavernosum. Furthermore, numbers of penile progenitor
cells were increased in DM ED rats.
Conclusions: Combined treatment of ESWT with SDF-1 eMSC treatment is more effective than by a
single therapy. It could be used as a potential and effective synergistic treatment for DM ED.

Keywords: Extracorporeal shockwave therapy (ESWT); mesenchymal stem cells (MSCs); erectile dysfunction (ED); streptozotocin diabetes; penis

Submitted Jan 26, 2021. Accepted for publication Apr 16, 2021.

doi: 10.21037/tau-21-79

View this article at: http://dx.doi.org/10.21037/tau-21-79

^ ORCID: 0000-0001-9930-0588. 


\section{Introduction}

It is common for diabetes mellitus (DM) patients to have erectile dysfunction (ED) (1) and ED decreases the quality of life in seriously (2). Since the prevalence of DM is increasing worldwide (3), ED cases in men who suffer from DM have increased sustainedly for decades $(4,5)$. The etiology of diabetes mellitus erectile dysfunction (DM ED) is thought to be due to vascular damage caused by hyperglycemia which induces the function of peripheral or autonomic nerve system (1). The main medication currently used for DM ED is a phosphodiesterase 5 inhibitor (PDE5I) that can induce vascularization to the corpus cavernosum (6). However, it is known that patients with DM have poor responses to PDE5I (7). Since erectile function plays essential role in the psychological wellbeing and reproductive function, an effective approach to treat diabetes DM ED is in urgent need.

Extracorporeal shockwave therapy (ESWT) has been applied for treating various disorders, including musculoskeletal disorders and myocardial infarction with damaged tissues $(8,9)$. Therapeutic ultrasound can induce VEGF expression, thereby stimulating angiogenesis (10). Since the first report of treatment of ED with Li-ESWT by Vardi et al. (11), numerous studies have reported effects of ESWT on DM ED (12-14). It has been suggested that ESWT can promote regeneration and tissue repair by stimulating angiogenesis and restoring blood flow to injured areas (15). ESWT can also upregulate the stromal cell derived factor-1 (SDF-1) expression, to recruit endothelial progenitor cells into damaged tissues $(16,17)$.

Mesenchymal stem cells (MSCs) are hopeful cell sources for regenerative medicine with extraordinary self-renewal abilities. In addition, they can transform into different types of cells (18). MSC therapy can promote cellular growth and prevent apoptosis. Thus, it can be used for treating nerve injury, trauma, inflammatory diseases, and so on (19).

A previous study has shown that ED in a rat model of cavernous nerve injury can be treated by injecting adiposederived stem cells overexpression stromal cell-derived factor-1 (SDF-1) (20). Yamaguchi et al. have also reported that locally delivered SDF-1 can improve vascularization by augmenting endothelial progenitor cells (EPCs) (21). SDF-1 expressing mesenchymal stem cells (SDF-1 eMSCs) can increase angiogenesis and improve ED by releasing chemokines that could help MSCs migrate to targeted tissues (22).

In this study, we made an ED streptozotocin (STZ)- induced DM rat model and then administered ESWT or SDF-1 eMSC treatment. We hypothesized that ESWT combined with SDF-1 eMSC therapy could enhance angiogenesis and regenerate nerve functions of corpus cavernosum and thereby accelerate the treatment for DM ED using a rat model. We evaluated the effect of such combination on DM ED and underlying mechanisms involved in such effect. We present the following article in accordance with the ARRIVE reporting checklist (available at http://dx.doi.org/10.21037/tau-21-79).

\section{Methods}

\section{Study design and experimental animals preparation}

250-300 g weighing fifty 8-week-old male Sprague-Dawley rats were purchased from Orient Bio Co. (Seongnam, Korea). At a dose of $65 \mathrm{mg} / \mathrm{kg}$ body weight, streptozotocin (STZ, Sigma-Aldrich chemical company, USA) was injected intraperitoneal to induced DM (23). Normal group of rats were injected with an equal amount of the solution ( $0.1 \mathrm{~mol} / \mathrm{L}$ citrate-phosphate buffer, $\mathrm{pH} 4.5)$ used to dissolve STZ. At $72 \mathrm{hrs}$ after injection, blood samples were obtained from tail veins. DM was diagnosed by blood glucose level above $300 \mathrm{mg} / \mathrm{dL}$. An Accu-Chek active blood glucose monitor (Hoffmann-La Roche Ltd., Basel, Switzerland) was used to determine blood glucose level. After 12 weeks, erectile function test was performed and normal erectile functioning rats were excluded. When seminal strain was not found in a week after putting adult female rat with a streptozotocin-induced DM rat together, this experimental rat was included for the next process. The rat was excluded when seminal stain appeared. Then rats were divided into five groups ( $\mathrm{n}=10$ per group): (I) Normal group (n=10, without STZ), (II) DM ED, (III) DM ED + ESWT group, (IV) DM ED + SDF-1 eMSC group, and (V) DM ED + ESWT + SDF-1 eMSC group. Experiments were performed under a project license (NO.: CUMC2016-0310-01) granted by the Institutional Animal Care and Use Committee of the Catholic University of Korea, in compliance with institutional guidelines for the care and use of animals.

\section{SDF-1-expressing MSCs}

The study was conducted in accordance with the Declaration of Helsinki (as revised in 2013). The culture of bone marrow mesenchymal stem cells (BM-MSCs) were 
done in Dulbecco's modified Eagle's medium (DMEM, Gibco, Gaithersburg, MD, USA) supplemented with $20 \%$ fetal bovine serum (FBS; Gibco) and $5 \mathrm{ng} / \mathrm{mL}$ basic fibroblast growth factor (bFGF; Cell Signaling Technology, Danvers, MA, USA) at $37{ }^{\circ} \mathrm{C}$ with $5 \% \mathrm{CO}_{2}$. Engineered BM-MSCs were cultured in 10\% FBS DME. To generate engineered BM-MSCs, SDF-1 genes, hTERT, C-myc, and tetracycline transactivator ( $\mathrm{t} T A$ ) were synthesized and applied using a pBD lentiviral vector (SL BIGEN, Seongnam, Korea). To obtain monoclonal cell population, antibiotics were used to select transfected engineered MSCs. Based on SDF-1 expression and cell proliferation, final monoclonal cells were selected. SDF-1-expressing engineered BM-MSCs (SDF-1 eMSCs) were irradiated just before in vivo injection.

The SDF-1 sequence was obtained from GenBank (accession number: NM_000609.6). DNA sequencing was performed for confirming the frame of introduced sequence (Cosmo Genetech, Seoul, Korea). The seeding of modified cells was done in a 12 -well plates, density of $1 \times 10^{5}$ cells per well. After 48 hours of cell culture, SDF-1 protein levels were measured using ELISA kits (R\&D Systems, Minneapolis, MN, USA) from the harvested supernatants. The SDF-1 expression level was about $10 \mathrm{ng} / \mathrm{mL}$.

\section{ESWT administration}

Under anesthesia, each rat was placed in the supine position and shaved and the prepuce was degloved. Ultrasound gel was applied to the penis and a shockwave applicator (CENOWAVE; HNT MEDICAL Co., Ltd., Seoul, Korea) was placed at the penis. A total of 300 shocks were delivered at an energy level of $0.1 \mathrm{~mJ} / \mathrm{mm}^{2}$ and a frequency of 120 shocks/min. This procedure was performed three times a week with one day break for four weeks, which is similar to the treatment course used for patients with ED in clinical practice (11).

\section{ESWT and SDF-1 eMSC treatment}

Except normal group, all rats in DM ED, DM ED + ESWT, DM ED + SDF-1 eMSC, and DM ED + ESWT + SDF-1 eMSC groups underwent bilateral intracavernous injections. After anesthetized with $2.0 \%$ isoflurane inhalation, rats were placed in a supine position with its lower abdomen shaved and the penis drawn out from the prepuce. To track the location of SDF-1 eMSCs, labeling MSCs with a fluorescent dye (CellTracker ${ }^{\mathrm{TM}}$ CM-DiI; Molecular
Probes, Eugene, OR, USA) was done before bilateral intracavernous injection of SDF-1 eMSCs. DM + SDF-1 eMSC and DM + ESWT + SDF-1 eMSC groups had bilateral intracavernous injection of $200 \mu \mathrm{L}$ PBS solution containing $1 \times 10^{6}$ SDF-1 eMSCs $(11,20)$. Just $200 \mu \mathrm{L}$ PBS bilateral intracavernous injection was done for rats in normal, DM, and DM + ESWT groups. SDF-1 eMSC injection was given one time per every week and repeated for four weeks. After these injections, rats in DM + ESWT and DM + ESWT + SDF-1 eMSC groups had Electrical Shock Wave Treatment. Under isoflurane anesthesia with a supine position, the penis was drawn out from the prepuce. A shock wave applicator was placed on the ultrasonic gel covered penis. According to previous studies $(12,24)$ each penis was treated with 300 impulses of ESWT at an energy flux density of $0.1 \mathrm{~mJ} / \mathrm{mm}^{2}$ with a frequency of $120 / \mathrm{min}$. With a break for one day, ESWT was treated three times per week, and repeated for four weeks.

\section{Measurement of Erectile Function}

All rats underwent erectile function test after receiving treatment for four weeks. Under general anesthesia, intracavernous pressure (ICP) and mean arterial pressure (MAP) was measured. Through a midline laparotomy, carotid artery and cavernous nerves were exposed. The corpus cavernosum was cannulated with a 25 -gauge butterfly needle filled with heparin $(250 \mathrm{U} / \mathrm{mL})$. A pressure transducer (Grass model S48 K; Astro-Med Inc., West Warwick, RI, USA) was connected to cannula and ICP was recorded. Then MAP was measured with A BD Intramedic PE-50 tubing (BD, Franklin Lakes, NJ, USA) inserted into carotid artery. Both ICP and MAP were recorded continuously $(20,25,26)$. After examining ED, rats were sacrificed by intraperitoneal injection of sodium pentobarbital $(50 \mathrm{mg} / \mathrm{kg})$ followed by bilateral thoracotomy. And the penis was harvested for western blot analysis and immunohistochemistry.

\section{Immunobistochemistry}

To prepare paraffin blocks, fresh penile midshaft tissues were harvested and fixed in $4 \%$ paraformaldehyde for $24 \mathrm{hrs}$ at $4^{\circ} \mathrm{C}$. Corpus cavernosum paraffin sections were immunofluorescence stained with neuron-specific $\beta$-III tubulin (diluted 1:200; Abcam, Cambridge, UK), stromal cell-derived factor-1 (SDF-1 diluted 1:200; Abcam, Cambridge, UK) and neuronal nitric oxide synthase (nNOS, 
diluted 1:200 Santa Cruz Biotechnologies, Santa Cruz, CA, USA). To stain nuclei, incubating with 4,6-diamidino-2phenylindole (DAPI; Vector Laboratories Inc., Burlingame, CA, USA) was performed. Digital images were obtained using a Zeiss LSM 510 Meta confocal microscope (Zeiss, Oberkochen, Germany). Mean intensity was then calculated using ZEN 2009 (Zeiss).

\section{Effect of ESWT in combination with SDF-1 eMSC treatment on ED in vivo}

$60 \mathrm{mg}$ of harvested penile tissues were immersed separately with $350 \mathrm{~mL}$ of $0.1 \mathrm{M} \mathrm{HCL}$ and homogenized in $2 \mathrm{~mL}$ lysate buffer (Precellys 24; Bertin Technologies, Montignyle-Bretonneux, France), which were then centrifuged at $14,000 \times \mathrm{g}$ for 10 minutes at $4{ }^{\circ} \mathrm{C}$. Supernatant was collected and a bicinchoninic acid (BCA) assay was performed to measure protein concentration in each sample. Cavernous cGMP levels and total NO level were calculated using a cGMP (cyclic guanosine monophosphate) direct immunoassay kit (K372, BioVision, San Francisco, USA). For last, Nitrate/Nitrite Fluorometric Assay Kit (Cayman Chemical) was used to calculate the total nitrate + nitrite concentration.

\section{Image and statistical analysis}

By using Image-J and Image-Pro Plus (Media Cybernetics, Silver Spring, MD, USA) the images of study's results were quantified. The statistical analysis was performed using SPSS 22.0 software (IBM, Armonk, NY, USA). Data are presented as mean \pm standard deviation (SD). Multigroup comparisons were made with ANOVA. Data expressed as proportions were assessed with a Chi-square test. Values of $\mathrm{P}<0.05$ were considered statistically significant difference.

\section{Results}

\section{ESWT + SDF-1 eMSC treatment can significantly improve DM ED}

Figure 1 shows the ICP results. Quantitative analysis results are shown in Figure $1 A$. The ICP was $0.69 \pm 0.13$ for the normal group, $0.27 \pm 0.08$ for the DM ED group, $0.41 \pm 0.06$ for the DM ED + ESWT group, $0.53 \pm 0.08$ for the DM $\mathrm{ED}+\mathrm{SDF}-1$ eMSC group, and $0.06 \pm 0.09$ for the DM ED + ESWT + SDF-1 eMSC group. Quantitative results of ICP were higher in treated groups than in the DM ED group. Thus, both ESWT and SDF-1 eMSC can definitely improve DM ED. The ICP/MAP ratio was significantly $(\mathrm{P}<0.05)$ higher in the DM ED + ESWT + SDF-1 eMSC group than in the DM ED group. It was closer to the ICP/ MAP ratio of the normal group than to any other treated group.

\section{ESWT + SDF-1 eMSCT improves smooth muscle contents in the corpus cavernosum}

As shown in Figure $2 A, \alpha$-smooth muscle actin ( $\alpha$-SMA) concentrations were elevated in treated groups. Figure $2 B$ shows quantitative results in each group. The DM ED + ESWT + SDF-1 eMSC group had the highest $\alpha$-SMA level, followed by the ED + SDF-1 eMSC group and the DM ED + ESWT group. Higher $\alpha$-SMA concentration in injured corpus cavernosum tissues means more positive influence on tissue repair. CM-Dil and DAPI also showed the highest concentration in groups treated with ESWT + SDF-1 eMSCs, meaning that ESWT could increase the quantity of SDF-1 eMSCs. In quantitative analysis, mean intensity of a-SMA was higher in ESWT or SDF-1 eMSC group than in the DM ED group $\left({ }^{*} \mathrm{P}<0.05\right)$. It was the highest in the ESWT + SDF-1 eMSC group $\left({ }^{\#} \mathrm{P}<0.01\right)$.

\section{ESWT can increase the intensity of SDF-1 expression in the corpus cavernosum}

As shown in Figure $3 A$, CM-Dil and SDF-1 showed the highest concentrations in the group treated with ESWT + SDF-1 eMSCs $\left({ }^{\#} \mathrm{P}<0.01\right)$. As shown in Figure 3B, SDF-1 expression level in ESWT +SDF-1 eMSC group was higher than that in the group treated with SDF-1 eMSC alone. It was even higher than that in the normal group. This result reveals that ESWT can increase SDF-1 expression significantly, which can help vascularization of the corpus cavernosum by recruiting endothelial progenitor cells (21).

\section{ESWT + SDF-1 eMSC treatment induces nNOS and NO/ cGMP expression in the corpus cavernosum}

Representative images of nNOS staining in the copus cavernosum are shown in Figure 4. Figure $4 A$ shows nNOS expression in each group. Figure $4 B$ shows quantitative results in each group. nNOS concentration was elevated in treated groups. It was the highest in the ESWT + SDF1 eMSC group. Which is essential for vasodilation of the 

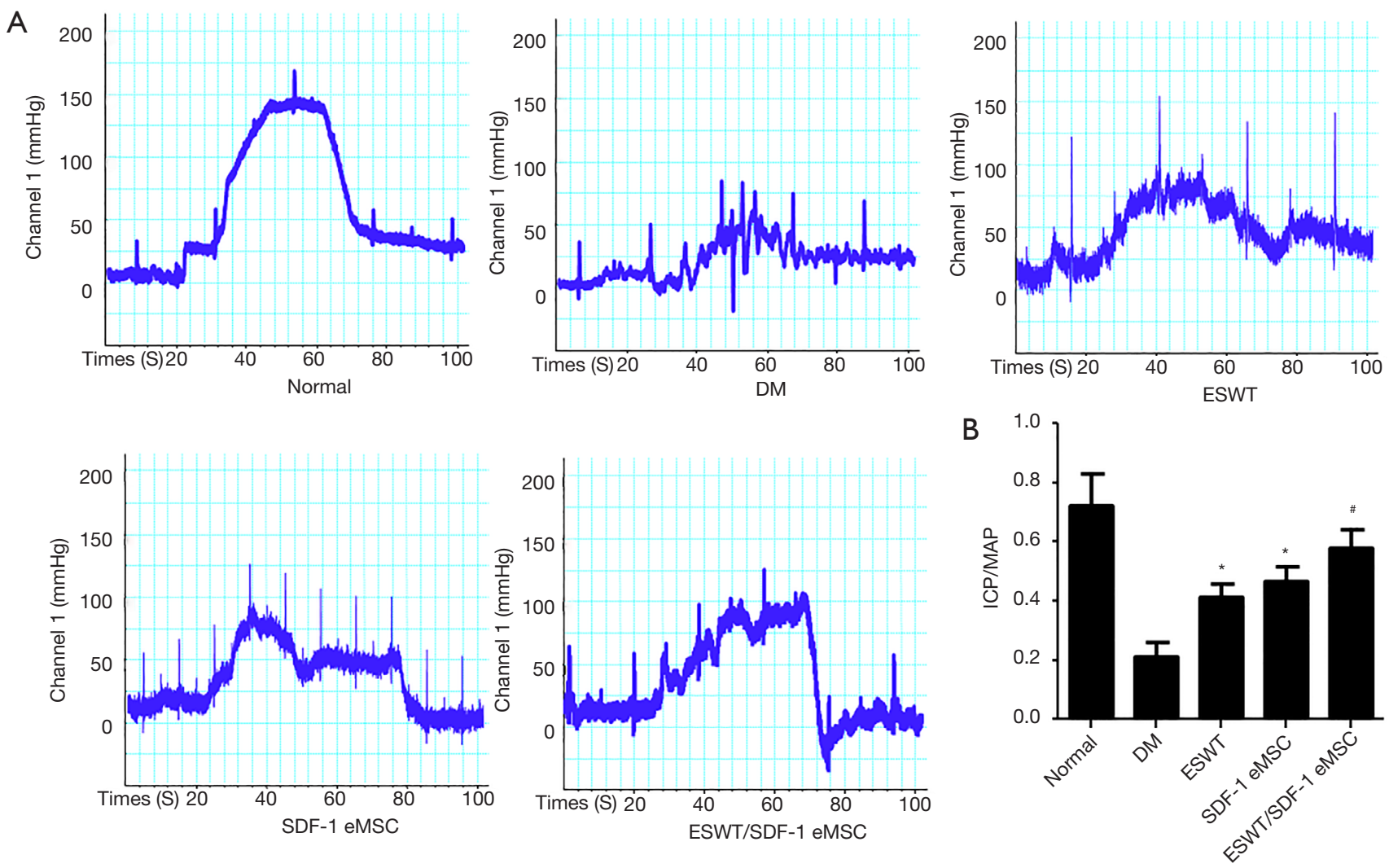

Figure 1 Comparison of erectile functions among groups. (A) Representative images of ICP in response to electrical stimulation of the cavernosal nerve. (B) Ratio of ICP to mean MAP of each group. Each bar shows the mean value (standard deviation). *, P value <0.05 compared to DM ED rats; ", P value <0.01 compared to DM ED rats.

corpus cavernosum. Figure $4 C$ shows that $\mathrm{NO}$ and cGMP levels in the ESWT + SDF-1 eMSC group were higher than in other groups. They were most close to those of the normal group $\left({ }^{\#} \mathrm{P}<0.01\right)$. Liu et al. have suggested that $\mathrm{NO}$ can mediate vasodilation in smooth muscle of vessels through the NO/cGMP pathway (27).

\section{ESWT + SDF-1 eMSC treatment increases penile progenitor cells in DM ED rats}

5-ethynyl-2-deoxyuridine (EdU) can be used for labeling and tracking of mesenchymal stromal cells (28). All rats had intraperitoneal injections of $50 \mathrm{mg} / \mathrm{kg}$ EdU before therapies. After four weeks, immunofluorescence staining was done to examine penile progenitor cells in the corpus cavernosum. As shown in Figure 5A, EdU positive cells were more prominent in the EWST + SDF-1 eMSC treated group and the ESWT group than those in the DM ED group and SDF-1 eMSC group. This means that ESWT can induce penile progenitor cells and enhance SDF-1 eMSCs in the corpus cavernosum. Quantitative results are shown in Figure 5B. There were more EdU positive cells in the ESWT group $\left({ }^{*} \mathrm{P}<0.05\right)$ and the ESWT + SDF-1 eMSC group $\left({ }^{\#} \mathrm{P}<0.01\right)$ than in other groups.

\section{Discussion}

The mechanism of DM ED is considered to be due to chronic hyperglycemia that causes blood vessel spasm and vascular injury such as veno-occlusive disorder in the corpus cavernosum $(23,24,29)$. Leading to insufficient blood supply and oxidative stress response (30). Oxidative stress response reduces endothelial nitric oxide synthase (eNOS) and nNOS in the corpus cavernosum by damaging neurocytes and endothelial cells, thus decreasing the concentration of NO known to cause DM ED (31). 

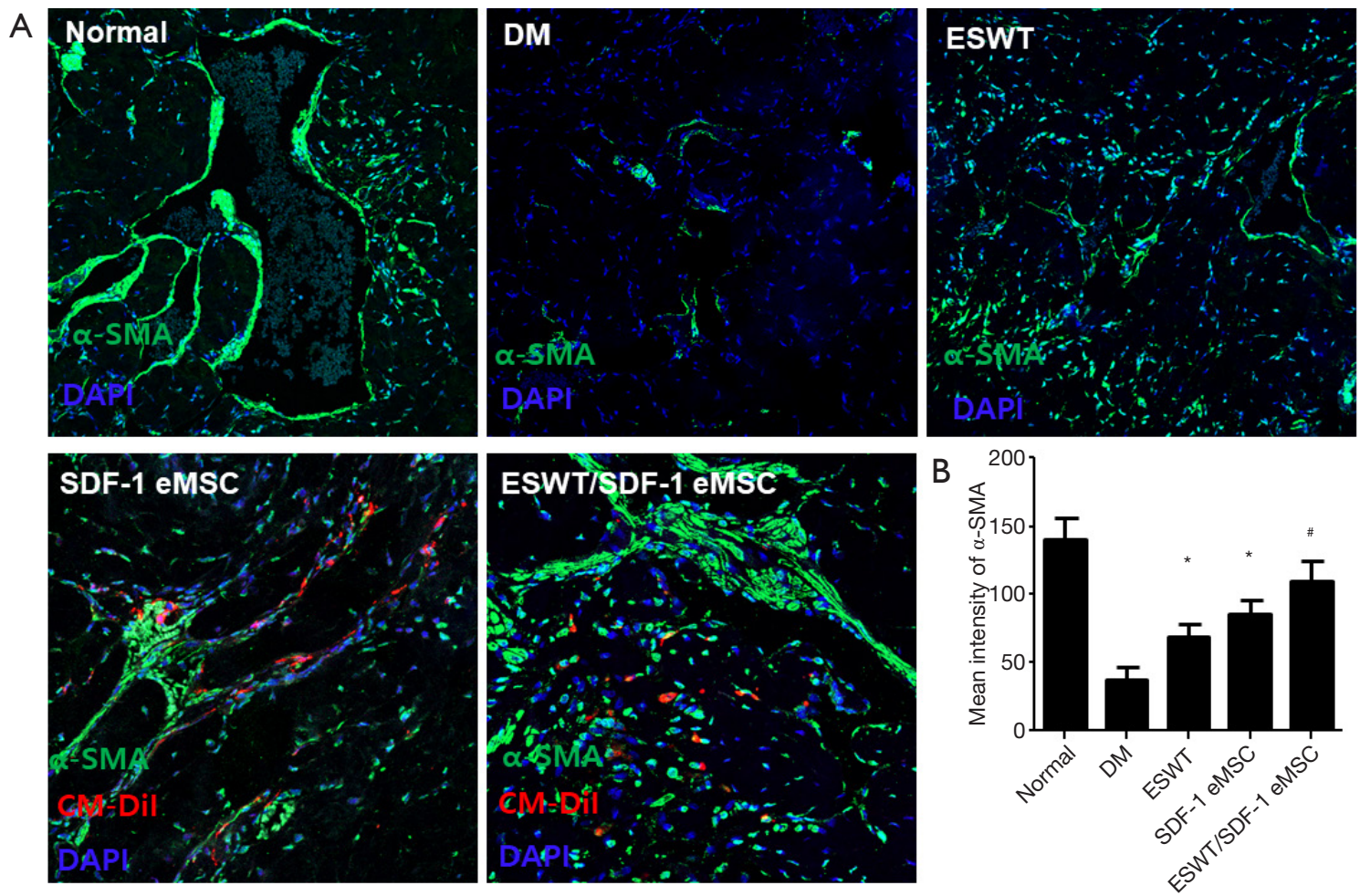

Figure 2 Comparison of smooth muscle contents among groups. (A) Representative images of immunofluorescenece stained corpus cavernosum after treatment with ESWT and SDF-1 MSC injection. Red (CM-Dil) indicates MSC; Green represents $\alpha$-SMA; Blue (DAPI) represents cell nuclei. Original magnification: $\times 200$; (B) mean intensity of $\alpha$-SMA in each group. Each bar shows the mean value (standard deviation). *, P value $<0.05$ compared to DM ED rats; ${ }^{*}$, P value $<0.01$ compared to DM ED rats.

ESWT and MSC have been used as treatment options for ED therapy (12,32-34). Several studies have proven that ESWT can promote angiogenesis and tissue proliferation. Lin et al. have found that a low intensity-ESWT can activate penile progenitor cells in situ by increasing angiogenesis and proliferation (35). Jeon et al. have shown that a combination of ESWT with a KH-204 herbal formulation can increase activation of antioxidant activity and penile progenitor cells in diabetic ED rats (17). One study has shown that ESWT can enhance the homing of MSCs into tissues of DM ED rats (13).

In our study, cell progenitor cells labelled with EdU positive cells were more prominent in the ESWT group and ESWT + SDF-1 eMSC group than in the DM ED group or the SDF-1 eMSC group. This means that ESWT can help induce penile progenitor cells in the corpus cavernosum. This results align with previous studies which proved ESWT enhancing activation of penile progenitor cell $(17,35)$. Cun et al. described that SDF-1 is a chemokine that plays critical role in homing of stem and progenitor cells (36). In our study SDF-1 eMSC treated group did show increase in penile progenitor cells but not significantly.

Also in this experiment, the quantity of SDF-1 expression in the ESWT + SDF-1 eMSC group was higher than that in the SDF-1 eMSC alone group. It was even higher than that in the normal group. This result shows that ESWT can increase SDF-1 expression significantly to help vascularization of the corpus cavernosum.

As adult multipotent stem cells, MSCs are easy to obtain from almost every tissue of the body (37). They have ability to differentiate into various types of cells such as muscle cells, neuron cells, and endothelial cells (38-40) essential for treating ED. A previous study has proven that MSC treatment can improve ED recovery by promoting cellular growth and prevent apoptosis of injured tissues $(22,41,42)$. Furthermore, during tissue repair and cell proliferation, SDF-1 levels are likely to be high (43) and it has been 

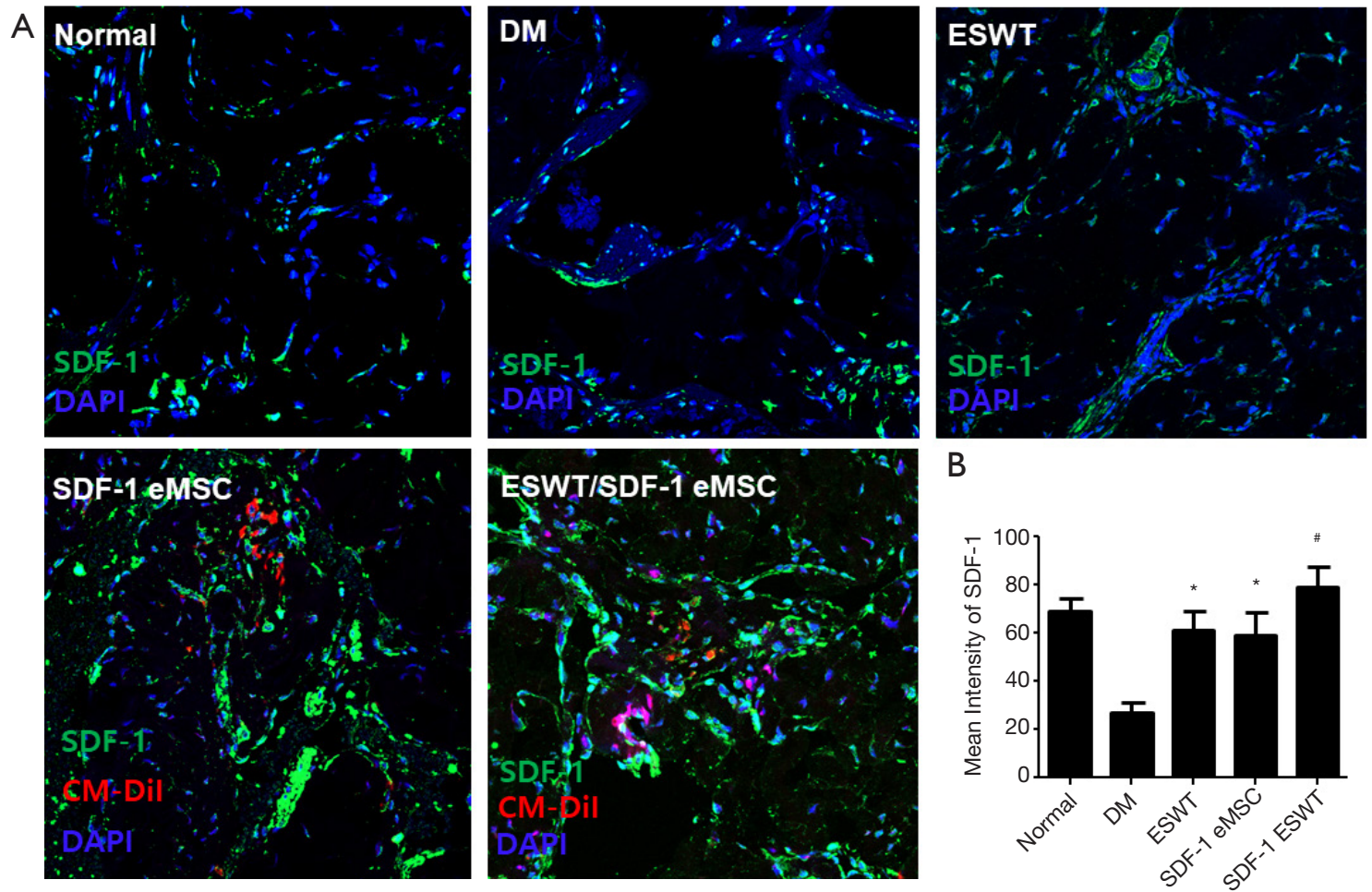

B

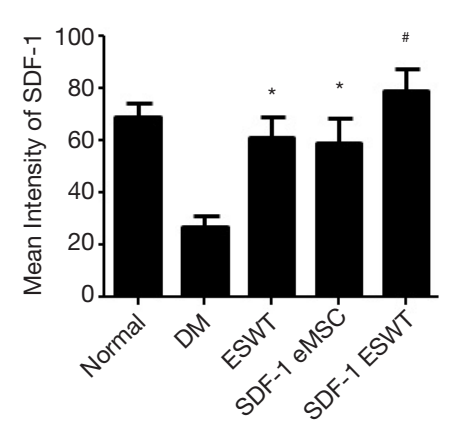

Figure 3 Comparison of SDF-1 expression among groups. (A) Representative images of immunofluorescence stained SDF-1 expression in the corpus cavernosum. Green indicates SDF-1; Red (CM-Dil) indicates MSC; Blue (DAPI) indicates cell nuclei. Original magnification: $\times 200$; (B) mean intensity of SDF-1. Each bar shows the mean value (standard deviation). *, P value $<0.05$ compared to DM ED rats; \#, P value $<0.01$ compared to DM ED rats.

proven that SDF-1 can accelerate angiogenesis by recruiting endothelial progenitor cells into injured tissues (21) and restore neuron cells by driving injected stem cells to injured nerves (20). A previous study has reported that BM-MSC expressing SDF-1 can express more SDF-1 than ordinary BM-MSC (22). Thus, in this study, we expected greater benefits by implanting SDF-1 expressing MSCs and used it for bilateral corpus cavernosum injections.

Zhu et al. have combined MSC treatment with ESWT and found that the combination of ESWT with MSC could improve ED better than a single treatment by expressing more VEGF (44). In this study, we used SDF-1 eMSC in combination with ESWT, expecting better outcome than ESWT with MSC treatment.

We found that the ICP/MAP ratio was higher in the DM $\mathrm{ED}+\mathrm{ESWT}+\mathrm{SDF}-1$ eMSC group than in the DM ED + ESWT or the ED + SDF-1 eMSC group. This indicates that a combination of ESWT with SDF-1 eMSC can be more efficient than a single therapy. Furthermore, the DM
$\mathrm{ED}+\mathrm{ESWT}+\mathrm{SDF}-1$ eMSC group showed significant increase in $\alpha$-smooth muscle actin ( $\alpha$-SMA) concentration, indicating that levels of smooth muscles in injured corpus cavernosum tissues were increased, thus having more positive influence on tissue repair than by ESWT or SDF-1 eMSC single treatment.

Oxidative stress due to vascular damage by hyperglycemia can cause neurocyte damage and reduce eNOS levels in the corpus cavernosum. Decreased NO level can induce ED (31). In the present study, NO and cGMP levels in the ESWT + SDF-1 eMSC group were higher than in other groups, which mediates vasodilation in smooth muscles of vessels to improve $\mathrm{ED}$.

STZ-induced DM ED rats are classed as type $1 \mathrm{DM}$ rats. However, only $10 \%$ of DM cases are type $1 \mathrm{DM}$ while $90 \%$ of cases are type $2 \mathrm{DM}$ in a clinical setting (45), which can be a limitation of this study.

In conclusion, for DM ED treatment, ESWT combined with SDF-1 eMSC treatment is more effective than 

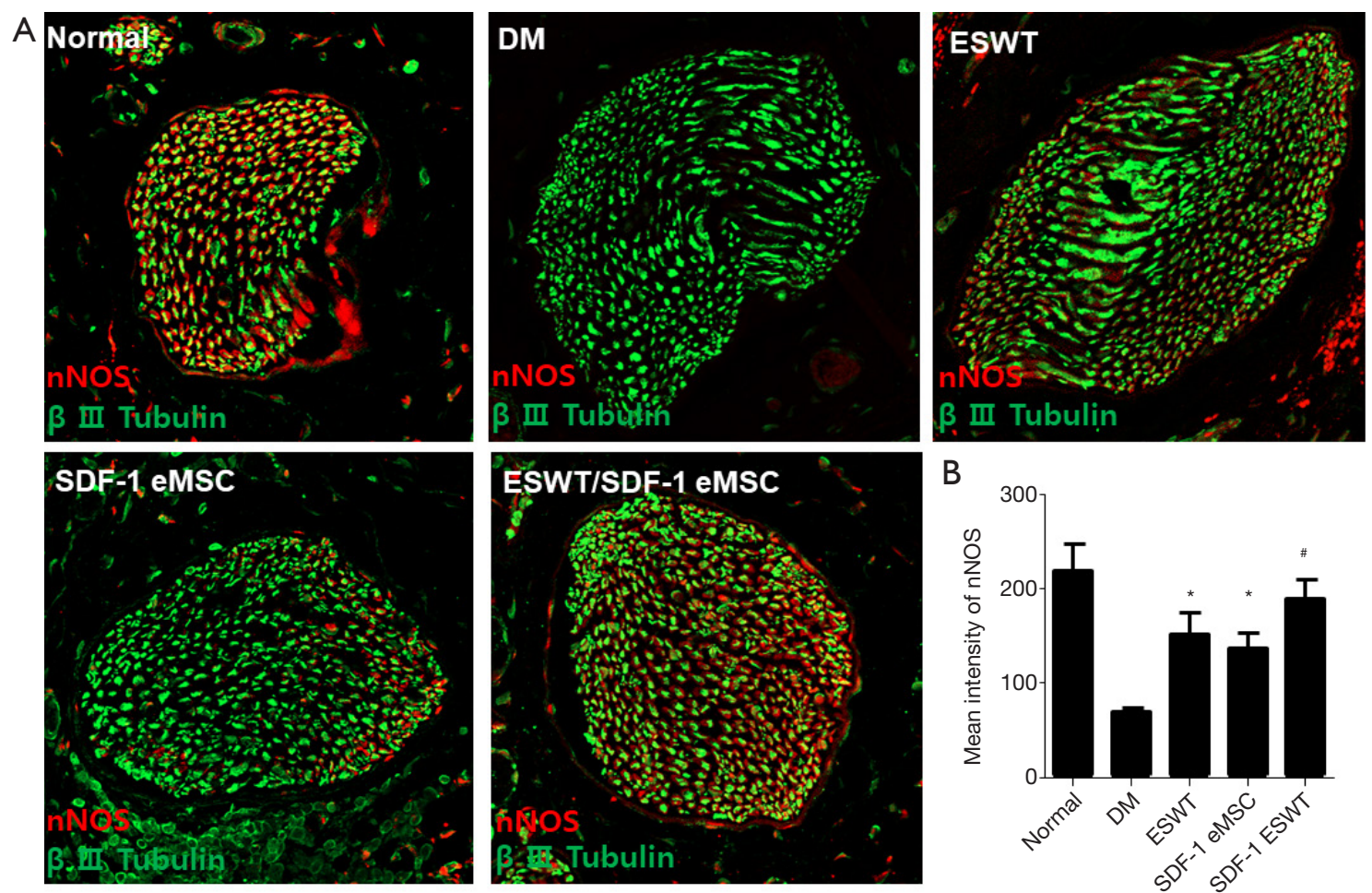

\section{B}
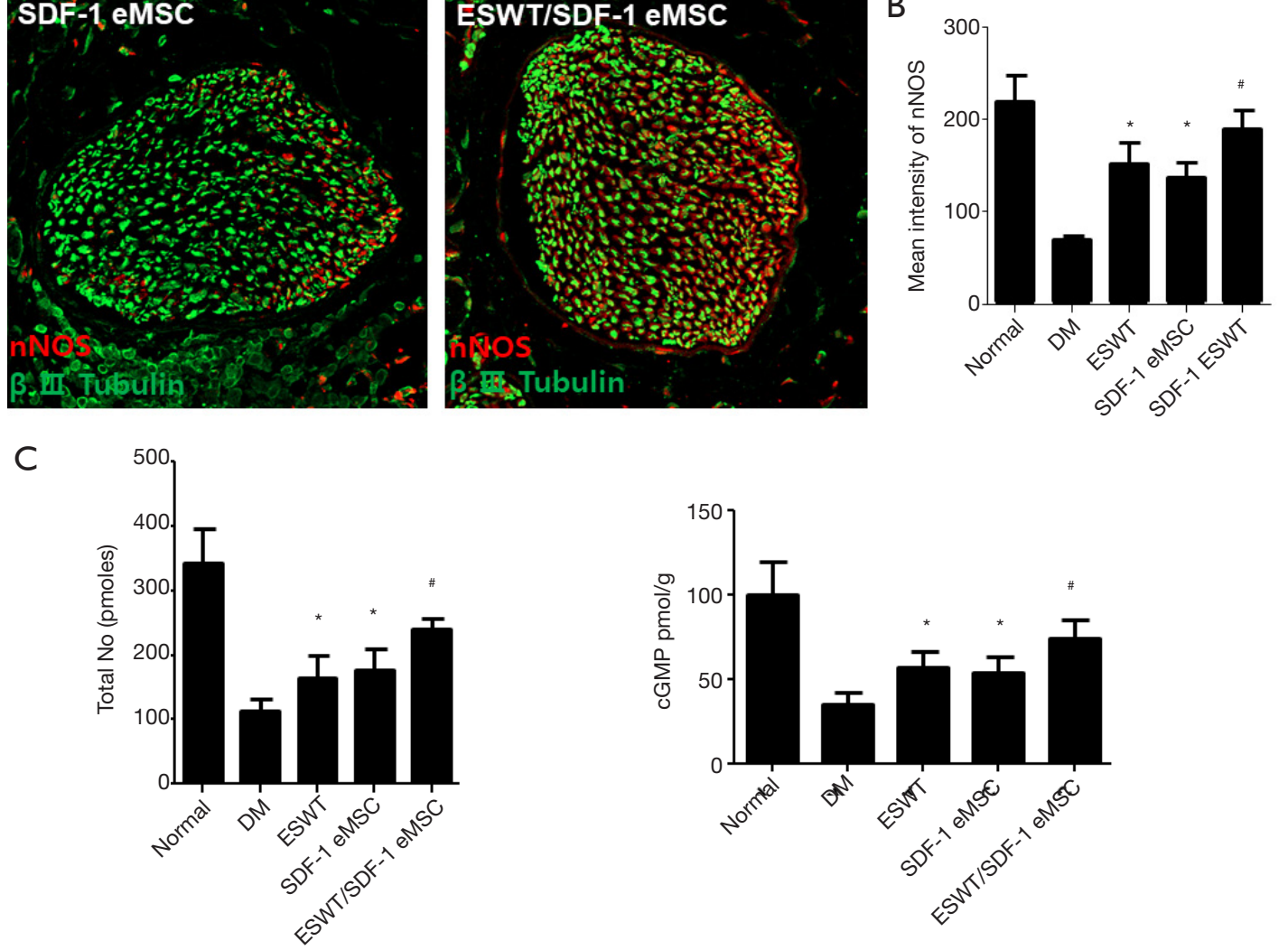

Figure 4 ESWT + SDF-1 eMSC treatment induces nNOS expression in the corpus cavernosum. (A) Representative nNOS staining images of the corpus cavernosum. Original magnification: $\times 200$. (B) The ratio of nNOS in each group. Each bar shows the mean value (standard deviation). (C) The ratio of total NO and cGMP in each group. Each bar shows the mean value (standard deviation). *, $\mathrm{P}$ value $<0.05$ compared to DM ED rats; ", P value $<0.01$ compared to DM ED rats.

using a single therapy. Such combination could be a potential and effective synergistic treatment for DM ED. Considering that EWST + SDF-1 eMSC therapy is a localized treatment, we believe that this therapy can help drugs penetrate into the corpus cavernosum and enhance absorption of drugs. Clinical trials are needed to determine whether additional oral medications or IV injections could enhance treatment efficacy in the future. 

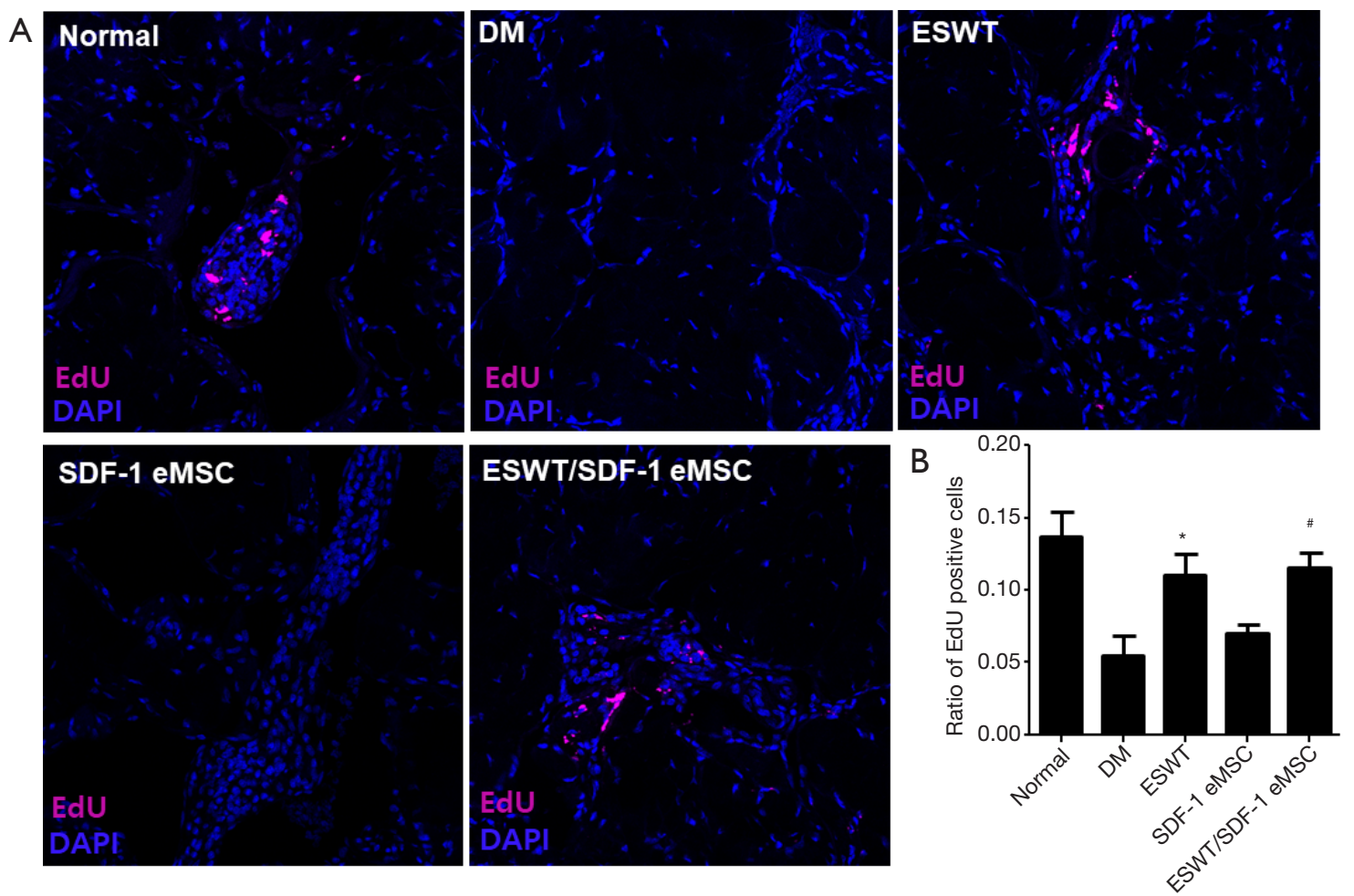

Figure 5 ESWT + SDF-1 eMSC treatment increases the number of penile progenitor cells in DM ED rats. (A) Pink indicates EdU (5-ethynyl-2-deoxyuridine) labelled penile progenitor cells and blue indicates DAPI stained nuclei. Original magnification: $\times 200$; (B) Quantitative results of EdU labelled cells in each group. Each bar shows the mean value (standard deviation). *, P value $<0.05$ compared to DM ED rats; ", P value $<0.01$ compared to DM ED rats.

\section{Acknowledgments}

Funding: This research was supported by Basic Science Research Program through the National Research Foundation of Korea (NRF) funded by the Ministry of Education (2020R1I1A1A01074732). Also supported by Research Fund of Seoul St. Mary's Hospital, The Catholic University of Korea (grant number : ZC20DESI0798).

\section{Footnote}

Reporting Checklist: The authors have completed the ARRIVE reporting checklist. Available at http://dx.doi. org/10.21037/tau-21-79

Data Sharing Statement: Available at http://dx.doi. org/10.21037/tau-21-79

Conflicts of Interest: All authors have completed the ICMJE uniform disclosure form (available at http://dx.doi. org/10.21037/tau-21-79). The authors have no conflicts of interest to declare.

Ethical Statement: The authors are accountable for all aspects of the work in ensuring that questions related to the accuracy or integrity of any part of the work are appropriately investigated and resolved. The study was conducted in accordance with the Declaration of Helsinki (as revised in 2013). Experiments were performed under a project license (NO.: CUMC-2016-0310-01) granted by the Institutional Animal Care and Use Committee of the Catholic University of Korea, in compliance with institutional guidelines for the care and use of animals.

Open Access Statement: This is an Open Access article distributed in accordance with the Creative Commons Attribution-NonCommercial-NoDerivs 4.0 International License (CC BY-NC-ND 4.0), which permits the noncommercial replication and distribution of the article with the strict proviso that no changes or edits are made and the 
original work is properly cited (including links to both the formal publication through the relevant DOI and the license). See: https://creativecommons.org/licenses/by-nc-nd/4.0/.

\section{References}

1. Isidro ML. Sexual dysfunction in men with type 2 diabetes. Postgrad Med J 2012;88:152-9.

2. De Berardis G, Franciosi M, Belfiglio M, et al. Erectile dysfunction and quality of life in type 2 diabetic patients: a serious problem too often overlooked. Diabetes Care 2002;25:284-91.

3. Wild S, Roglic G, Green A, et al. Global prevalence of diabetes: estimates for the year 2000 and projections for 2030. Diabetes Care 2004;27:1047-53.

4. Bacon CG, Hu FB, Giovannucci E, et al. Association of type and duration of diabetes with erectile dysfunction in a large cohort of men. Diabetes Care 2002;25:1458-63.

5. Cheng JYW, Ng EML, Ko JSN, et al. Physical activity and erectile dysfunction: meta-analysis of population-based studies. Int J Impot Res 2007;19:245-52.

6. Peak TC, Anaissie J, Hellstrom WJG. Current perspectives on stem cell therapy for erectile dysfunction. Sex Med Rev 2016;4:247-56.

7. Martínez-Jabaloyas JM, Gil-Salom M, Villamón-Fort R, et al. Prognostic factors for response to sildenafil in patients with erectile dysfunction. Eur Urol 2001;40:6416; discussion 647.

8. Zissler A, Steinbacher P, Zimmermann R, et al. Extracorporeal shock wave therapy accelerates regeneration after acute skeletal muscle injury. Am J Sports Med 2017;45:676-84.

9. Becker M, Goetzenich A, Roehl AB, et al. Myocardial effects of local shock wave therapy in a Langendorff model. Ultrasonics 2014;54:131-6.

10. Young SR, Dyson M. The effect of therapeutic ultrasound on angiogenesis. Ultrasound Med Biol 1990;16:261-9.

11. Vardi Y, Appel B, Jacob G, et al. Can low-intensity extracorporeal shockwave therapy improve erectile function? A 6-month follow-up pilot study in patients with organic erectile dysfunction. Eur Urol 2010;58:243-8.

12. Behr-Roussel D, Giuliano F. Low-energy shock wave therapy ameliorates erectile dysfunction in a pelvic neurovascular injuries rat model. Transl Androl Urol 2016;5:977-9.

13. Qiu X, Lin G, Xin Z, et al. Effects of low-energy shockwave therapy on the erectile function and tissue of a diabetic rat model. J Sex Med 2013;10:738-46.
14. Clavijo RI, Kohn TP, Kohn JR, et al. Effects of lowintensity extracorporeal shockwave therapy on erectile dysfunction: a systematic review and meta-analysis. J Sex Med 2017;14:27-35.

15. Wang B, Ning H, Reed-Maldonado AB, et al. Lowintensity extracorporeal shock wave therapy enhances brain-derived neurotrophic factor expression through PERK/ATF4 signaling pathway. Int J Mol Sci 2017;18:433.

16. Aicher A, Heeschen C, Sasaki KI, et al. Low-energy shock wave for enhancing recruitment of endothelial progenitor cells: a new modality to increase efficacy of cell therapy in chronic hind limb ischemia. Circulation 2006;114:2823-30.

17. Jeon SH, Bae WJ, Zhu GQ, et al. Combined treatment with extracorporeal shockwaves therapy and an herbal formulation for activation of penile progenitor cells and antioxidant activity in diabetic erectile dysfunction. Transl Androl Urol 2020;9:416.

18. Bunpetch V, Wu H, Zhang S, et al. From "bench to bedside": current advancement on large-scale production of mesenchymal stem cells. Stem Cells Dev 2017;26:1662-73.

19. Uccelli A, Moretta L, Pistoia V. Mesenchymal stem cells in health and disease. Nat Rev Immunol 2008;8:726-36.

20. Fandel TM, Albersen M, Lin G, et al. Recruitment of intracavernously injected adipose-derived stem cells to the major pelvic ganglion improves erectile function in a rat model of cavernous nerve injury. Eur Urol 2012;61:201-10.

21. Yamaguchi J, Kusano KF, Masuo O, et al. Stromal cell-derived factor-1 effects on ex vivo expanded endothelial progenitor cell recruitment for ischemic neovascularization. Circulation 2003;107:1322-8.

22. Jeon SH, Zhu GQ, Bae WJ, et al. Engineered mesenchymal stem cells expressing stromal cell-derived factor-1 improve erectile dysfunction in streptozotocininduced diabetic rats. Int J Mol Sci 2018;19:3730.

23. Wang ZH, Shang YY, Zhang S, et al. Silence of TRIB3 suppresses atherosclerosis and stabilizes plaques in diabetic ApoE-/-/LDL receptor-/- mice. Diabetes 2012;61:463-73.

24. Liu J, Zhou F, Li GY, et al. Evaluation of the effect of different doses of low energy shock wave therapy on the erectile function of streptozotocin (STZ)-induced diabetic rats. Int J Mol Sci 2013;14:10661-73.

25. Qiu X, Fandel TM, Ferretti L, et al. Both immediate and delayed intracavernous injection of autologous adiposederived stromal vascular fraction enhances recovery of erectile function in a rat model of cavernous nerve injury. 
Eur Urol 2012;62:720-7.

26. Albersen M, Fandel TM, Zhang H, et al. Pentoxifylline promotes recovery of erectile function in a rat model of postprostatectomy erectile dysfunction. Eur Urol 2011;59:286-96.

27. Liu X, El-Mahdy MA, Boslett J, et al. Cytoglobin regulates blood pressure and vascular tone through nitric oxide metabolism in the vascular wall. Nat Commun 2017;8:14807.

28. Lin G, Huang YC, Shindel AW, et al. Labeling and tracking of mesenchymal stromal cells with EdU. Cytotherapy 2009;11:864-73.

29. Hidalgo-Tamola J, Chitaley K. Review type 2 diabetes mellitus and erectile dysfunction. J Sex Med 2009;6:916-26.

30. Yin GN, Park SH, Song KM, et al. Establishment of in vitro model of erectile dysfunction for the study of highglucose-induced angiopathy and neuropathy. Andrology 2017;5:327-35.

31. Burnett AL, Lowenstein CJ, Bredt DS, et al. Nitric oxide: a physiologic mediator of penile erection. Science 1992;257:401-3.

32. Vardi Y, Appel B, Kilchevsky A, et al. Does low intensity extracorporeal shock wave therapy have a physiological effect on erectile function? Short-term results of a randomized, double-blind, sham controlled study. J Urol 2012;187:1769-75.

33. Chen Z, Han X, Ouyang X, et al. Transplantation of induced pluripotent stem cell-derived mesenchymal stem cells improved erectile dysfunction induced by cavernous nerve injury. Theranostics 2019;9:6354-68.

34. Wang X, Liu C, Xu Y, et al. Combination of mesenchymal stem cell injection with icariin for the treatment of diabetes-associated erectile dysfunction. PLoS One 2017;12:e0174145.

35. Lin G, Reed-Maldonado AB, Wang B, et al. In situ activation of penile progenitor cells with lowintensity extracorporeal shockwave therapy. J Sex Med 2017;14:493-501.

36. Cun Y, Diao B, Zhang Z, et al. Role of the stromal cell

Cite this article as: Shin D, Jeon SH, Tian WJ, Kwon EB, Kim GE, Bae WJ, Cho HJ, Hong SH, Lee JY, Kim SW. Extracorporeal shock wave therapy combined with engineered mesenchymal stem cells expressing stromal cell-derived factor-1 can improve erectile dysfunction in streptozotocin-induced diabetic rats. Transl Androl Urol 2021;10(6):2362-2372. doi: $10.21037 /$ tau-21-79 derived factor-1 in the biological functions of endothelial progenitor cells and its underlying mechanisms. Exp Ther Med 2021;21:39.

37. Philippart P, Meuleman N, Stamatopoulos B, et al. In vivo production of mesenchymal stromal cells after injection of autologous platelet-rich plasma activated by recombinant human soluble tissue factor in the bone marrow of healthy volunteers. Tissue Eng Part A 2014;20:160-70.

38. Müller P, Lemcke H, David R. Stem cell therapy in heart diseases-cell types, mechanisms and improvement strategies. Cell Physiol Biochem 2018;48:2607-55.

39. Morikawa S, Mabuchi Y, Kubota Y, et al. Prospective identification, isolation, and systemic transplantation of multipotent mesenchymal stem cells in murine bone marrow. J Exp Med 2009;206:2483-96.

40. Blondheim NR, Levy YS, Ben-Zur T, et al. Human mesenchymal stem cells express neural genes, suggesting a neural predisposition. Stem Cells Dev 2006;15:141-64.

41. Mangir N, Turkeri L. Stem cell therapies in postprostatectomy erectile dysfunction: a critical review. Can J Urol 2017;24:8609-19.

42. Bochinski D, Lin GT, Nunes L, et al. The effect of neural embryonic stem cell therapy in a rat model of cavernosal nerve injury. BJU Int 2004;94:904-9.

43. Liang X, Huang X, Zhou Y, et al. Mechanical stretching promotes skin tissue regeneration via enhancing mesenchymal stem cell homing and transdifferentiation. Stem Cells Transl Med 2016;5:960-9.

44. Zhu GQ, Jeon SH, Bae WJ, et al. Efficient promotion of autophagy and angiogenesis using mesenchymal stem cell therapy enhanced by the low-energy shock waves in the treatment of erectile dysfunction. Stem Cells Int 2018;2018:1302672.

45. Danaei G, Finucane MM, Lu Y, et al. National, regional, and global trends in fasting plasma glucose and diabetes prevalence since 1980: systematic analysis of health examination surveys and epidemiological studies with 370 country-years and 2.7 million participants. Lancet 2011;378:31-40. 